\title{
White Dwarfs
}

Peter Thejll

Niels Bohr Institute, Blegdamsvej 17, DK-2100 Copenhagen, Denmark

\section{White dwarfs as the end product of most stellar evolution}

It is the intention of this review to explain what white dwarfs are and why it is interesting to study them, and why the $\mathrm{H}_{2}^{+}$molecule is of special interest.

The evolution, from start to finish, of a star of mass less than about 2 solar masses $\left(\mathrm{M}_{\odot}\right)$, can roughly be summarized as follows:

- A cloud of gas contracts from the interstellar medium until hydrogen ignites at the center and a main sequence (MS) star forms. $\mathrm{H}$ is transformed to $\mathrm{He}$ and the MS phase continues until $\mathrm{H}$ is exhausted in the stellar core.

- H continues burning in a shell outside the He core while the core contracts. He "ashes" are added to the core, and a red giant star is formed as the envelope expands. The star evolves up the Red Giant Branch (RGB) (i.e. it becomes more and more luminous and the surface cools).

- Towards the end of the RGB phase, mass-loss from the upper layers increases until helium to carbon burning in the core ignites suddenly under degenerate conditions - this is called the Helium Flash (HF). The HF terminates the RGB evolution, and therefore also the mass-loss and the growth of the stellar core.

- The star readjusts its structure and the He-core burns steadily on the horizontal branch (HB) (a phase of nearly-constant luminosity) until fuel is exhausted in the He-core.

- Then the $\mathrm{C} / \mathrm{O}$ core contracts anew and the expansion of the envelope, and the growth of the core, during He-shell burning, mimics RGB evolution but relatively little mass is added to the core this time.

- The second ascent of the giant branch (the so-called Asymptotic Giant Branch, or AGB) continues with increased mass loss towards the end

- Rapid detachment of a considerable fraction of the remaining envelope and the hot core takes place, sometimes observable as the Planetary Nebulae (PN) phase.

- The PN is dispersed as the core contracts to a white dwarf (WD). 
- The WD cools for a long time, as internal kinetic energy and latent heat is released.

For stars of mass above $2 \mathrm{M}_{\odot}$ the evolution is different and will not be touched on here. A good, thorough introduction to stellar evolution can be found in Collins (1989) Kippenbahn \& Weigert (1991).

White dwarfs represent the end-stage of evolution for $90 \%$ of all stars. The remaining $10 \%$ evolve into neutron stars or black holes; they undergo rapid MS evolution followed by a supernova explosion. Because most stars end up as white dwarfs one can learn much about stellar evolution history by studying them the origins of WD properties are to be sought in the preceding evolution stages, as well as in basic physics.

The equation of state for degenerate matter determines when the ignition of the degenerate helium core takes place: this event essentially sets the mass of white dwarfs - if the flash is delayed the core grows larger and if the flash is early a smaller He-core is formed (Jørgensen \& Thejll 1993). Also, mass-loss increases towards the end of the RGB ascent so that the point of termination of the ascent sets upper limits to the eventual WD envelope mass. The mass-loss events that follow during the AGB ascent and during the PN phase trim the envelope mass to its final WD value. The nature of the mass-loss mechanisms is not well understood, but empirical fits to observations of red giant star massloss (RGB stars and AGB stars) exist (Reimers 1977, and Niuewenhuijzen \& de Jaeger 1990).

Residual burning of the H-shell in the upper layers throughout the evolution also deplete the amount of hydrogen left. Theory predicts that something like $10^{-4} \mathrm{M}_{\odot}$ of $\mathrm{H}$ will be left when the star finishes its AGB and PN phases. Thus, the total mass and detailed structure (envelope-mass, core mass, chemical composition of the layers, etc.) of white dwarfs depend on the physics of degenerate electrons, nuclear forces (fusion), atomic physics (opacities) and thermodynamics (structure, and conditions for convection).

After the PN has been dispersed the hot bare core of $\mathrm{C} / \mathrm{O}$ contracts until the electrons are degenerate yet again and no further contraction is possible. Cooling for the rest of the evolution of the Universe follows. This means that $W D$ cooling can be used to measure the age of the star-forming regions of the Galaxy. Cooling is rapid at first (e.g. tables of D'Antona \& Mazzitelli 1990), with the temperature reaching half its maximum value in just one million years, but as the object cools the rate of cooling slows down. In about $10^{10}$ years it is expected that $3000 \mathrm{~K}$ will be reached - in other words: it may be possible to find white dwarfs as old as the Galaxy itself. This method of measuring the age of the Galaxy (Wood 1992) is an important alternative to the other methods such as globular cluster chronology. No important nuclear reactions take place in white dwarfs once the degenerate state has been reached so the only source of energy for the flow of radiation is kinetic energy of the ions, and latent heat released in the crystallization of the ions that occurs towards the end. The rate of WD cooling is governed by the transparency of the surface layers. The interior is isothermal and the rate at which radiation leaks through the photosphere is 
set by the opacity. As the cooling rate slows down with lower $T_{\text {eff }}$, the WDs spend most of their life while cool, i.e. where molecular opacities are important - that is why it is worthwhile investing significant efforts in calculating the best possible molecular opacities for white dwarfs.

\section{White dwarf characteristics}

One important characteristic of white dwarfs is that the majority have more or less the same mass - 30\% of the DAs have masses between 0.5 and 0.55 $\mathrm{M}_{\odot}$ (Bergeron et al. 1992). As stars of masses from near $1 \mathrm{M}_{\odot}$ up to about 8 $\mathrm{M}_{\odot}$ all manage to become white dwarfs it is clear that mass-loss is needed in the preceding stages - and mass loss that is carefully tailored so as not to be too large nor too small.

The radiation we can see from white dwarfs comes from the photosphere - a layer only about $500 \mathrm{~m}$ thick. By analyzing spectra it is possible to determine $\mathrm{T}_{\text {eff }}, \log (g)$ and the composition - using line profiles and/or photometry. At an early stage of WD research it became evident that WDs fall into 2 major groups: Those with $\mathrm{H}$ dominated photospheres (75\% of the WDs), called DAs, and those dominated by $\mathrm{He}$, called DBs, plus a few intermediary cases (DABs and DBAs) as well as stars contaminated by elements heavier than He (DQs: showing $\mathrm{C}$ and/or $\mathrm{C}_{2}$ features, DZs: showing $\mathrm{Ca}$ and other "metal" features), and the DC that have only very faint depressions in their continua.

Explaining the origins of the abundance differences is a major goal of WD research as a successful explanation may have important bearing on earlier evolution stages: Because the gravitational field is so large, elements of different mass separate quickly, $\mathrm{H}$ floating on top of He etc. - this means that a DB may not contain any $\mathrm{H}$ at all and that perhaps such a lack can be explained by events in earlier stages of evolution where layers of the envelope was shed on the RGB, AGB and in the PN phase. Other possible explanations for this problem are discussed and reviewed by Shipman $(1988,1992)$.

As the WDs become cold it is observed that the relative abundance of DAs decreases - i.e. that photospheric $H$ is absent. This could mean that any $H$ present in cooling DAs is mixed with underlying $\mathrm{He}$ in a process governed by the efficiency of convection in $\mathrm{He}$ and the layer thickness of $\mathrm{H}$ left from the previous evolutionary stages - but unless the layer of $H$ is thinner than about $10^{-10} \mathbf{M}_{\odot}$, convective mixing cannot "mix away" the $\mathrm{H}$. This is an important clue to the previous stages of stellar evolution: Mass loss before the WD stage is so tuned that the condition for the maximum H-layer thickness is met in some cool DA WDs.

Further clues to the internal structure of WDs can be had from observing oscillations of WDs. Astereoseismology interprets non-radial g-mode pulsations (Bradley 1993), seen on the stellar surface as brightness variations. Such analyses have the potential for revealing the stellar mass, the interior structure, the rotation of the star and the rate of cooling which is related to the composition of the core, and are thus important tools for studying the interior of WDs. 
DBs as well as DAs oscillate, as do the much hotter PG1159-class objects (Winget et al. 1985). The oscillations are driven by mechanisms in which the opacity of the gas is coupled to its ionization-state so that the opacity depends on the pressure and temperature and these in turn depend on the radiation field. In $\mathrm{H}$ rich envelopes the oscillations take place near $10,000 \mathrm{~K}$, while the instability strip lies somewhere between 20,000 to $25,000 \mathrm{~K}$ in the He-rich DBs, and even higher for oxygen (the PG1159's). Oscillations become feasible when a convection zone has properties suitable for sustaining the cycle of partial ionization in each of these elements. Therefore the physics of convection becomes linked to the study of oscillations.

This coupling has been utilized in attempts to test current convection theories, such as the mixing-length approach (Fontaine 1984). Such theories contain rather arbitrarily chosen parameters that in principle can be tuned by requiring them to have such values that the predicted temperatures of instability strips coincide with the observed temperatures. Here accurate knowledge of stellar photosphere opacities enter the picture, because accurate temperatures can only be had from observations if the opacities that are used for calculating line profiles and continuum fluxes are accurate. One study of the accurate modelling of DB photospheres, and the impacts on mixing length theory calibrations, is that by Thejll et al. (1991).

For the DAs one important source of opacity, until recently not well modelled, is the ultraviolet absorption of $\mathrm{H}_{2}$ and $\mathrm{H}_{2}^{+}$. In the DAs surrounding the DA instability strip this molecule shows strong absorption features near the Lyman$\alpha$ line - so-called satellites of Lyman- $\alpha$. At the relevant temperatures the flux in DAs drops very quickly on the blue side of Lyman- $\alpha$ anyway but the flux dominates the luminosity of the stars in the range from Lyman- $\alpha$ into the 2000$3000 \AA$ range. Figure 1 shows an example of such an object, observed with the International Ultraviolet Explorer satellite.

If we understand cool white dwarf opacities well we have a better chance of calculating their cooling rate, and hence the age of the Galaxy by counting the space density of cool white dwarfs. This gives one of the strongest motivations for studying molecular opacities in white dwarfs.

The presence of molecules in white dwarf photospheres is not always just determined by having the appropriate temperature range for the existence of the molecules in question. An example are the DQ WDs - these are helium rich cool white dwarfs (i.e. DBs) that have a convection zone in the envelope that reaches so deep into the stellar interior that the diffusion-distributed tail of the central carbon core is "dredged-up" to the photosphere and becomes visible at temperatures from $15,000 \mathrm{~K}$ and down (e.g. Thejll et al. 1990). At these temperatures carbon shows CI and CII lines as well as - at the lower temperatures - molecular $\mathrm{C}_{2}$ bands, such as the Swan bands, which also must be modelled under the extreme pressure conditions present in WDs.

Magnetic fields are observed in some, mainly cool $\left(\mathrm{T}_{\text {eff }}<10000 \mathrm{~K}\right)$, white dwarfs and the effects on atomic as well as molecular absorption features must then be included in the analysis - see Bues \& Karl (1994). Bues (1991) discusses 

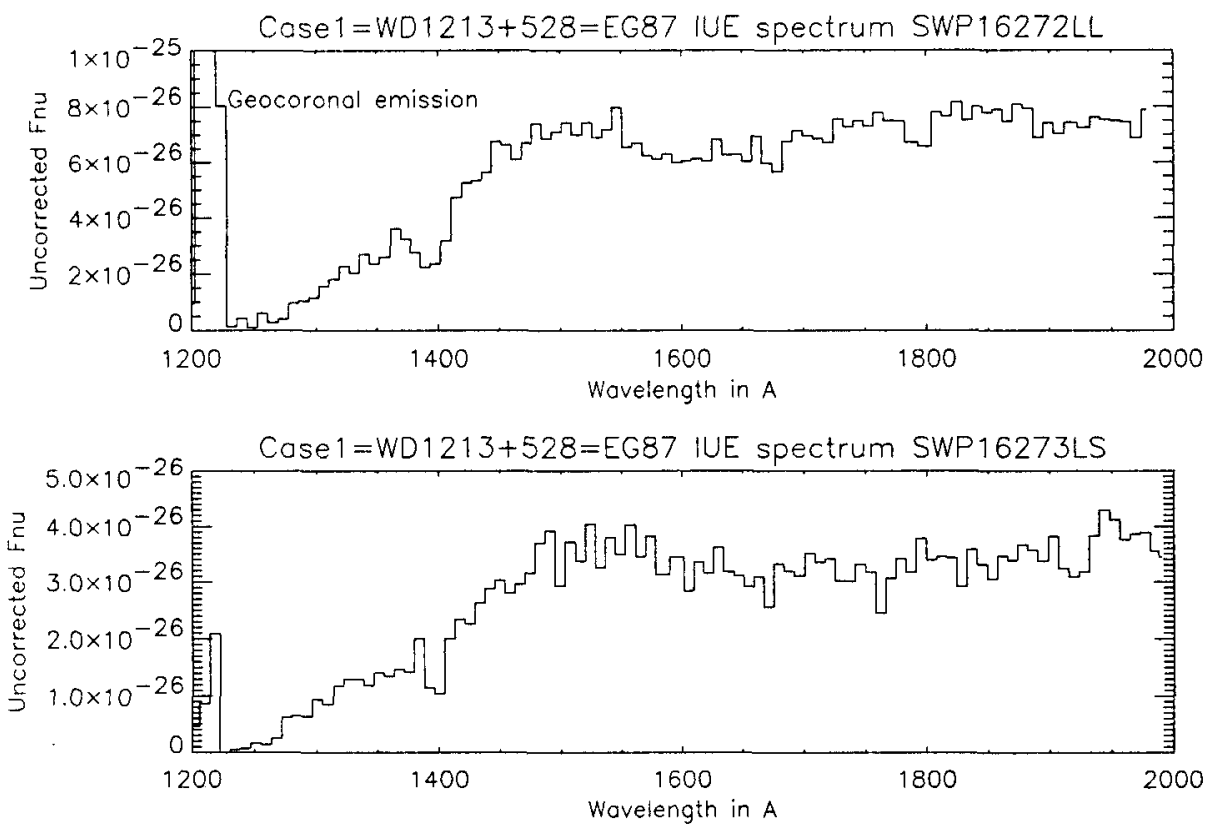

Fig. 1. Examples of ultraviolet spectra, observed by Sion et al. (1984) of a DA white dwarf showing $\mathrm{H}_{2}^{+}$absorption near $1400 \AA$. The DA is Case 1 and the figure headers gives other names as well as the numbers of the two IUE spectra shown. The upper spectrum was taken using the large aperture of the IUE camera and the lower with the small, hence the differences in the size of the geocoronal emission feature in the center of the Lyman- $\alpha$-line. The spectra have been smoothed for display purposes, and some of the non-photospheric features removed, such as cosmic ray hits. The spectra have not been otherwise corrected for instrument artifacts or interstellar reddening. It seems that only the $1400 \AA$ feature is clearly present. Other features, near $1600 \AA$ and $2000 \AA$, due to $\mathrm{H}_{2}$ and higher systems have been identified by Allard \& Koester (1992) in this object, but very careful reductions of the data are required to bring out these fainter features with certainty.

the WD G99-37 which shows strong $\mathrm{C}_{2}$ and $\mathrm{CH}$ absorbtion features as well as CI lines. Strong molecular features from other species are rare in WDs, and the non-observability of $\mathrm{CN}$ and $\mathrm{CH}$ features are typically used to set upper limits to element abundances (e.g. Zeidler-K.T. et al. 1986).

Strong features from "metals" such as $\mathrm{Ca}, \mathrm{Mg}, \mathrm{Si}$ and Fe in DZ WDs become more and more apparent as the stars cool, perhaps because of accretion onto cool stars from the interstellar medium - as the radiation pressure decreases 
accretion becomes possible. Zeidler-K.T. et al. (1986) shows spectra and analyses of typical DZ WDs.

At low temperatures it is also possible to form collision induced systems like $\mathrm{H}_{2}-\mathrm{H}_{2}$ and $\mathrm{H}_{2}-\mathrm{He}$ that have continuum opacities (see Borysow this volume). Borysow \& Frommhold (1989 and references therein) have studied such opacities and they are important for the modelling of cool white dwarfs where all the information about $\mathrm{T}_{\text {eff }}$, and $\log (g)$ may have to be gleaned from such things as the shape of the continuum - there being no, or only few, line-forming elements left in the atmosphere.

\section{The $\mathrm{H}_{2}^{+}$molecule}

The opportunity to understand the interior of white dwarfs better gives the motivation for studying any opacity that is important near one of the instability strips. For the case of the oscillating DBs one opacity-related problem was the inconsistency that existed between the UV-flux based $\mathrm{T}_{\text {eff }}$ determinations and those based on analysis of optical line profiles of HeI. This discrepancy has been cleared up (Thejll et al. 1991). For the cool DAs and the oscillating DAs (called "ZZ Ceti" stars) the focus is on the $\mathrm{H}_{2}$ and $\mathrm{H}_{2}^{+}$molecules and the special absorption features present near the maximum-flux region of the spectra. Because these features are strong and are in the maximum-flux region they represent the largest source of opacity that, until recently, was not well understood. The reason uncertain opacities are of concern to interpreters of spectra is not just that regions of the spectrum may not be analyzable, but that strong features block the radiation and cause back-warming of the atmosphere so that the structure of the photosphere is changed and the form of the spectrum in other regions change and may become unanalyzable, in the worst cases. Being strong, the UV features of $\mathrm{H}_{2}, \mathrm{H}_{2}^{+}$, and also $\mathrm{H}_{3}^{+}$, therefore require understanding for satisfactory modelling of cool DAs and ZZ Ceti stars.

These "quasi-molecules", formed during atom-atom or atom-ion collisions, give rise to line-like features, called satellites of the Lyman- $\alpha$ line, predicted theoretically in the 1970's and observed first, outside laboratories, in the 1980's by the IUE satellite in DA WDs (Greenstein 1980). Stewart et al. (1973) calculated crossections of the satellites due to $\mathrm{H}_{2}^{+}$, using nearest-neighbour, quasi-staticapproximations and this work was used to identify the features at $1400 \AA$ in IUE spectra by Nelan \& Wegner (1985), Holm (1985) and Koester (1985), simultaneously. At temperatures above $15000 \mathrm{~K}$ the $\mathrm{H}_{2}^{+}$satellite at $1400 \AA$ is stronger than the feature at $1600 \AA$ due to H-H collisions. Sando \& Wormhoudt (1973) calculated classically the expected absorption of the $\mathrm{H}_{2}$ feature. While both the Stewart et al. (1973) and the Sando \& Wormhoudt (1973) work served well to identify the features they did not give satisfactory fits to real spectra.

Allard et al. (1991) have lately modelled the many Lyman- $\alpha$ satellites in a comprehensive study of atom-atom and ion-atom interactions for binary, and higher, systems, and today this work (Allard \& Koester 1992) gives the best fits to observed DA spectra. The work rests on the use of square-well potentials 
(Allard 1978), which permits analytical calculation of the correlation function, and an appropriate density expansion that allows for the inclusion of effects in the far wings, which are important for the broad Lyman- $\alpha$ line.

Museth et al. (1994) have recently calculated full non-adiabatic quantum mechanical expressions for the crossection of $\mathrm{H}_{2}^{+}$avoiding some of the simplifications of the work by Allard et al. (1991).

\section{Summary}

I have described how WDs fit in to the stellar evolution picture and what questions they may help answer - e.g. the details of nucleosynthesis, mass-loss in the giant stages, and the age of the Galaxy. Notably astereoseismology of WDs can give information about internal structure to such a high degree of accuracy that understanding the "satellite" UV absorption features near Lyman- $\alpha$ becomes of great importance for bringing into agreement the photospheric analysis, and the things revealed by $\mathrm{ZZ}$ Ceti analysis. Recent advances in the modelling of $\mathrm{H}_{2}^{+}$, and other quasi-molecular absorbers, have the potentials for achieving this in the near future.

For those seeking further insight into the state of the art in white dwarf research, the biennial proceedings of the WD workshops can be recommended: Wegner (1988), Vauclair \& Sion (1990) and Barstow (1992).

Acknowledgments. Research support from the Carlsberg Foundation is gratefully acknowledged.

\section{References}

Allard N., 1978, J. Phys. B., 11, 1383

Allard N., Kielkopf J., 1991, Astron. Astrophys., 242, 133

Allard N., Koester D., 1992, Astron. Astrophys., 258, 464

Barstow M. (ed.), White Dwarfs, 1992, Kluwer Academic, NATO ASI Series vol. 403

Bergeron P., Saffer R., Liebert J., 1992, Astrophys. J., 394, 228

Borysow A., Frommhold L., 1989, Astrophys. J., 341, 549

Bradley P.A., Winget D.E., Wood M.A., 1993, Astrophys. J., 406, 661

Bues I., 1991, In: G. Vauclair, E.M. Sion (eds.), White Dwarfs, Kluwer Academic, NATO ASI Series vol. 336

Bues I., Karl L., 1994 In: P.Thejll \& U.G.Jørgensen (eds.), Poster Session Proceedings of IAU Coll. 146, Copenhagen University, p. 20

Collins G.,W. II, 1989, The fundamentals of stellar astrophysics, W.H. Freeman Co., New York

D'Antona F., Mazzitelli I., 1990, Annual Reviews of Astronomy and Astrophysics, 28, 139

Fontaine G. Tassoul M., Wesemael F., 1984, Proc. of the 25th Liege International Astrophysical Colloquium, Liege: Université de Liege, p. 328

Greenstein J., 1980, Astrophys. J., 241, L89 
Holm A.V., Panek R.J., Schiffer III F.H., Bond H.E., Kemper E., Grauer A.D., 1985, Astrophys. J., 289, 774

Jørgensen U.G., Thejll P., 1993, Astron. Astrophys., 272, 255

Kippenbahn R., Weigert A., 1991, Stellar Structure and Evolution, Springer Verlag A\&A Library, Berlin

Koester D., Weidemann V., Zeidler-K.T. E.-M., Vauclair G., 1985, Astron. Astrophys., 142, L5

Museth K., Linderberg J., Billing G., Thejll P., 1994, In: P.Thejll \& U.G.Jørgensen (eds.), Poster Session Proceedings of IAU Coll. 146, Copenhagen University, p. 82

Nelan E.P., Wegner G., 1985, Astrophys. J., 289, L31

Niuewenhuijzen H., and de Jaeger C., 1990, Astron. Astrophys., 231, 134

Reimers D., 1977, Astron. Astrophys. , 61, 217

Sando K., Wormhoudt J.C., 1973, Phys. Rev. A., 7, 1889

Shipman H. L., 1988, In G. Wegner (ed.), White Dwarfs, p. 220, Springer Verlag: LNP 328

Shipman H. L., 1992, In M. Barstow, White Dwarfs, p. 555, Kluwer Academic, NATO ASI Series, vol. 403

Sion E.M., Wesemael F., Guinan E.F., 1984, Astrophys. J., 279, 758

Stewart J.C., Peek J.M., Cooper J., 1973, Astrophys. J., 179, 983

Thejll P.A., Shipman H.L., MacDonald. J., 1990, Astrophys. J., 361, 197

Thejll P., Vennes S., Shipman H.L., 1991 Astrophys. J., 370, 355

Vauclair G., Sion. E.M., (ed.) 1990, White Dwarfs, Kluwer Academic, NATO ASI Series vol. 336

Wegner G. (ed.), 1988, White Dwarfs, Springer Verlag, Berlin, Lecture Notes in Physics vol. 328

Winget D.E., Kepler S.O., Robinson E.L., Nather R.E., D. O'Donoghue, 1985, Astrophys. J., 292, 606

Wood M. A., 1992, Astrophys. J., 386, 539

Zeidler-K.T. E.-M., Weidemann V., Koester D., 1986, Astron. Astrophys., 155, 356 